\title{
Changes in the Pattern of Primary Hyperparathyroidism in Czech Republic
}

\author{
Petr Broulík', Svatopluk Adámek ${ }^{2}$, Petr Libánský ${ }^{1}$, Jozef Kubinyi ${ }^{3}$ \\ ${ }^{1} 3^{\text {rd }}$ Department of Medicine - Department of Endocrinology and Metabolism, \\ First Faculty of Medicine, Charles University in Prague and General University \\ Hospital in Prague, Prague, Czech Republic; \\ ${ }^{2} 3^{\text {rd }}$ Department of Surgery, First Faculty of Medicine, Charles University in Prague \\ and University Hospital Motol, Prague, Czech Republic; \\ ${ }^{3}$ Institute of Nuclear Medicine, First Faculty of Medicine, Charles University \\ in Prague and General University Hospital in Prague, Prague, Czech Republic \\ Received February 16, 2015; Accepted June 1, 2015.
}

Key words: Primary hyperparathyroidism - Calcemia - Osteodystrophy - iPTH Nefrolithiasis - Asymptomatic PHPT

\begin{abstract}
Primary hyperparathyroidism (PHPT) is currently the most common endocrine disorder in Czech Republic after diabetes and thyroid diseases particularly in postmenopausal women. Over the past 40 years PHPT has changed from a rare severe disease of the bones and kidneys to common disease with hypertension, peptic ulcer, pancreatitis, easy fatigue and proximal muscle weakness. During 43 years we have examined one of the greatest groups of patients with PHPT. In the early 1970 the estimated incidence of PHPT in former Czechoslovakia was approximately 8 cases per 100000 persons per year. Our data showed that the incidence of PHPT increased sharply to 24 cases per 100000 persons per year in same community with the introduction of automated serum calcium and iPTH measurement. The disease is four times more frequent in women as in man. The ratio women to men did not changed since 1981. However the incidence of PHPT changed in Czech Republic from previous years, it develops around the fifth decade of life and is increasingly discovered with advancing age. The incidence of hypertension, diabetes mellitus, cholelithiasis, pancreatitis and peptic ulcer among patients with PHPT is higher as compared with the incidence of these diseases in the general population. However there are still patients suffering from bone and renal complication of PHPT. Removing the adenoma by an experienced surgeon is the first choice of treatment of patients with PHPT.The study offers valuable data on the actual state of hyperparathyroid patients in the Czech Republic.
\end{abstract}

Mailing Address: Prof. Petr Broulík, MD., DSc., $3^{\text {rd }}$ Department of Medicine Department of Endocrinology and Metabolism, First Faculty of Medicine, Charles University in Prague and General University Hospital in Prague, U Nemocnice 1, 12808 Prague 2, Czech Republic; e-mail: pbrou@lf1.cuni.cz 


\section{Introduction}

Primary hyperparathyroidism (PHPT) is currently the most common endocrine disorder after diabetes and thyroid diseases particularly in postmenopausal women. The clinical presentation of PHPT has dramatically changed in the last 40 years. The disease is now characterized by mild hypercalcemia, mild glandular enlargement and less traditional classic bone and kidney manifestations (Adami et al., 2002). Up to about 40 years ago in former Czechoslovakia as well as in other Western countries the disease was rarely diagnosed. The change in clinical presentation was largely due to the introduction of automated serum calcium and iPTH (intact parathyroid hormone) measurement. PHPT mainly affects middle-aged adults, peaks in the seventh decade and occurs more frequently in females than in males. The incidence of PHPT has changed dramatically. In the early 1970 the estimated incidence of PHPT in former Czechoslovakia was approximately 8 cases per 100000 persons per year. Our data showed that the incidence of PHPT increased sharply to 24 cases per 100000 persons per year in same community with the introduction of automated serum calcium and iPTH measurement. Earlier PHPT was considered a rare illness characterized by bone disease and urinary calculi. Many new clinical features, such as hypertension, mental disturbances, myopathy, peptic ulcer disease, pancreatitis and cholelithiasis have been added to the clinical spectrum of PHPT. Primary hyperparathyroidism is a relatively common endocrine disorder which in 80 percent of cases is caused by a single adenoma arising in one of the four parathyroid glands.

Most cases are now asymptomatic and usually detected on routine biochemical screening. Despite the absence of classical features, even asymptomatic primary hyperparathyroidism is known to affect the skeleton. It has been reported that patients suffering from symptomatic PHPT have increased mortality before and after parathyroidectomy (Kiernan et al., 2006). This increase in mortality seems to be mainly due to an overrepresentation of cardiovascular death (Feig et al., 2008). Untreated PHPT cause endothelial dysfunction, left ventricular hypertrophy, cardiac rhythm disturbances and arterial hypertension. In 1970 in $3^{\text {rd }}$ Department of Medicine, First Faculty of Medicine, Charles University in Prague a group of clinicians, biochemists and surgeons has been established. This group up to now examined and successfully operated more than 2,000 patients. Based on our large file of 1,750 patients suffering from PHPT this review summarized pathological conditions associated with PTH (parathyroid hormone) excess. The individual study presented later in this paper has been drawn from this group.

\section{Material and Methods}

Study subjects

The whole series comprise 1,750 patients $(1,350$ female and 400 males aged 20-76) with biochemically, surgically and histologically proved PHPT during a period of 43 years (1970-2013) at the $3^{\text {rd }}$ Department of Medicine, First Faculty of 
Medicine, Charles University in Prague. The individual study presented later in this paper has been drawn from this group.

The diagnosis of PHPT was confirmed by histopathological diagnosis based on combination of different variables, including size and histological pattern of the excised glands. Off the 1,750 patients who underwent parathyroidectomy 1,630 had an adenoma (chief-cells) and 120 patients had hyperplasia. None of the 1,750 patients with PHPT had multiple endocrine neoplasias. The control group was drawn from 2,520 patients receiving periodic health examination at outpatient ward in $3^{\text {rd }}$ Department of Medicine, First Faculty of Medicine, Charles University in Prague from January 2004 to December 2005. The control group was carefully selected so that each control was matched with individual patients with regard to sex, and similar age. The following exclusion criteria were used: impaired renal function, malignant and secondary hypertension and myocardial infarction. All patients of the control group have been normocalcemic and with normal iPTH.

\section{Study protocol}

The standardized baseline screening included complete history, physical examination, skeletal radiographs, later since 1993 sonography of the abdomen, electrocardiogram and DEXA (dual energy X-ray absorptiometry).

\section{Laboratory methods}

Serum concentration of calcium, uric acid, glucose, cholesterol and creatinine were determined by standard laboratory techniques in the same serum specimen in each patient. Normal reference values were: calcium $2.15-2.65 \mathrm{mmol} / \mathrm{l}$, creatinine 44-105 $\mu \mathrm{mol} / \mathrm{l}$, and uric acid 140-340 $\mu \mathrm{mol} / \mathrm{l}$. The biochemical criteria for renal functional impairment included the level of serum creatinine greater than $110 \mu \mathrm{mol} / \mathrm{l}$. Serum intact PTH was assayed using immunoradiometric assay of intact PTH (16-65 pg/ml) and finally by two site immunochemiluminometric assay (Bayer Corp Chiron, Halstead, UK) mean values 1.6-6.9 pmol/l from 1995. Up to this date we have used PTH-RIA-100 Kit IRE Belgium measuring $C$ terminal PTH (normal values $1.00 \pm 0.30 \mu \mathrm{g} / \mathrm{l})$.

X-rays films were taken of the hands, skull and kidneys to look for bone disease and renal calculi or nephrocalcinosis. Since 1993 also ultrasound examinations were taken of the kidneys and gallbladder to look for calculi.

\section{Clinical evaluation}

The examination consisted of a physician completed questionnaire, which included questions whether at any time in the past the patient had received treatment for high blood pressure (BP) or had hypertension diagnosed and whether they were currently taking antihypertensive medication. 
Criteria for the diabetes mellitus were fasting glucose $>5.6 \mathrm{mmol} / \mathrm{l}$ with positive oGGT test (oral glucose tolerance test) or previously diagnosed type 2 diabetes mellitus.

Height and body weight were measured with participants standing without shoes and heavy outer garments. Body mass index (BMI) was calculated as weight divided by height squared $\left(\mathrm{kg} / \mathrm{m}^{2}\right)$ as a measure of relative weight. Obesity was defined as $\mathrm{BMI}>30 \mathrm{~kg} / \mathrm{m}^{2}$ for both sexes.

The study was done in accordance with the principles of the Helsinki Declaration and the procedures followed were in accordance with institutional guidelines. The study was approved by the Hospital Ethics Committee and all patients gave written informed consent.

Statistical and discriminant analysis of the laboratory tests were performed by analysis of variance followed by the multiple range test. All mean values are presented with one standard deviation (SD). A $\mathrm{P}<0.05$ (two sided test) was accepted as level of significance. The difference in percentages in both groups was tested using chi-square test.

\section{Results}

The patients with primary hyperparathyroidism had a significantly higher median level of iPTH measured by different methods (Table 1). The incidence of PHPT in Czech Republic increases from the fifth to the seventh decade of life and the number of cases peaks in elderly women (Figure 1). 40 years ago in former Czechoslovakia the PHPT mostly occurred in middle aged postmenopausal women.

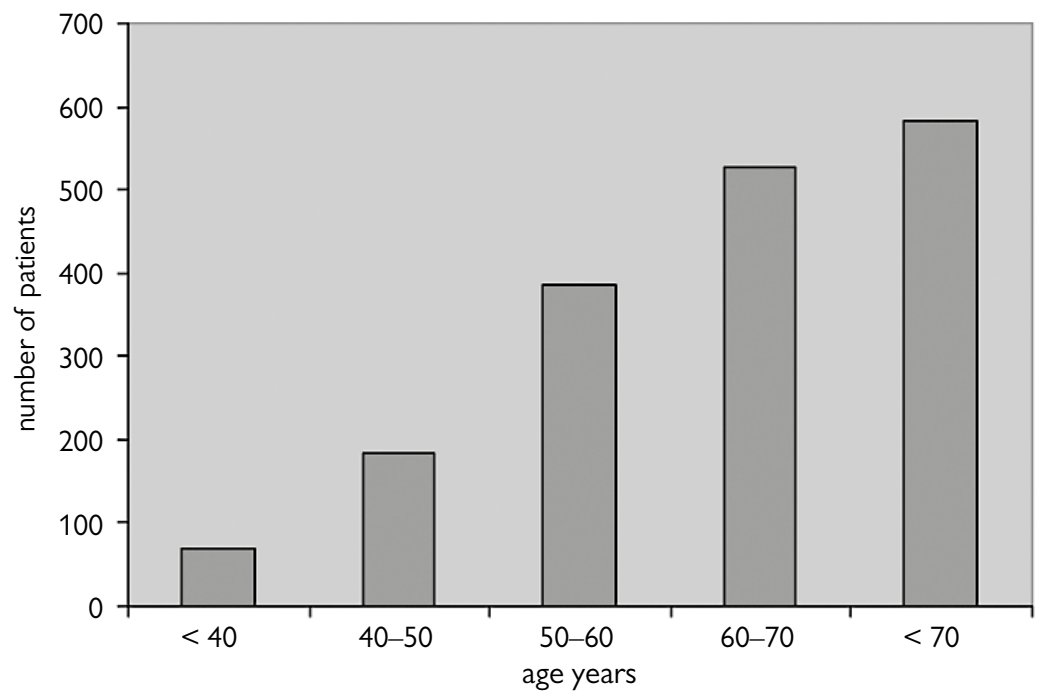

Figure 1 - Age distribution of 1,750 patients with primary hyperparathyroidism (PHPT) at the $3^{\text {rd }}$ Department of Medicine, First Faculty of Medicine, Charles University in Prague. 


\section{Table 1 - Clinical and biochemical data from 1,750 patients} with primary hyperparathyroidism (PHPT) (means \pm SD)

\begin{tabular}{lcc}
\hline & PHPT & Control \\
\cline { 2 - 3 } & $(\mathrm{N}=1,750)$ & $(\mathrm{N}=1,750)$ \\
\hline Sex (female/male) & $1,350 / 400$ & $1,350 / 400$ \\
Age (years) & $58 \pm 14$ & $60 \pm 15$ \\
BMI $\left(\mathrm{kg} / \mathrm{m}^{2}\right)$ & $32.8 \pm 2.6^{*}$ & $27.1 \pm 3.0$ \\
Serum calcium (mmol/l) & $3.01 \pm 0.1^{*}$ & $2.40 \pm 0.3$ \\
Serum phosphate $(\mathrm{mmol} / \mathrm{l})$ & $0.72 \pm 0.1 *$ & $1.10 \pm 0.2$ \\
Serum PTH (pmol/l) $(\mathrm{N}=1,459)$ & $16.9 \pm 2.8^{*}$ & $4.7 \pm 0.6$ \\
Uric acid (mmol/l) & $368 \pm 45^{*}$ & $266 \pm 42$ \\
Serum creatinin $(\mu \mathrm{mol} / \mathrm{l})$ & $74 \pm 5$ & $71 \pm 9$ \\
Serum total cholesterol (mmol/l) & $6.23 \pm 0.6$ & $6.4 \pm 0.3$ \\
\hline Diabetes mellitus (\%) & $24.00 \% *$ & $7.00 \%$ \\
Hypertension (\%) & $69.80 \% *$ & $47.90 \%$ \\
PHPT osteodystrophy (\%) & $36.00 \% *$ & $0.00 \%$ \\
Cholelithiasis (\%) & $28.89 \% *$ & $15.60 \%$ \\
Pancreatitis (\%) & $4.85 \% *$ & $1.15 \%$ \\
Nephrolithiasis (\%) & $31.40 \% *$ & $7.23 \%$ \\
Gastric and duodenal ulcer (\%) & $15.28 \% *$ & $4.30 \%$ \\
\hline
\end{tabular}

$* P<0.01$

At operation single adenoma formation was present in 1,630 patients, whereas diffuse hyperplasia was found in 120 other patients. 24 of the adenomas were found in an atypical anatomical localization. Parathyroid carcinoma was present in only five patients.

Classic symptoms of PHPT in Czech Republic are less common today but nephrolithiasis and bone involvement are still higher than in European countries. Kidney involvement due either to deposition of calcium in the renal parenchyma or to nephrolithiasis was present in $31.4 \%$ of our patients with PHPT and only $7.23 \%$ in control group.

The bone manifestation of hyperparathyroidism is hyperparathyroid osteodystrophy which occurred in $36.0 \%$ of our patients (Table 1 ). The most common changes include resorption of the phalangeal tuft and replacement of the usually sharp cortical outline of the bone in the digits by an irregular outline (subperiosteal resorption), generalized decalcification, bone cysts and the so called brown tumors. Also low bone mineral density particularly at site enriched in cortical bone.

Our patients with hyperparathyroidism often have articular symptoms involving the knees, shoulders, and hands. Rheumatic symptoms develop in approximately half of all our patients with PHPT.

In the individual study hypertension was documented preoperatively in 726 patients with PHPT (69.8\%) whilst 294 of the patients were normotensive. The blood pressure recorded in 726 subjects with hypertension averaged 
$150 \pm 3.8 \mathrm{~mm} \mathrm{Hg}$ (systolic) and $97.0 \pm 3.0 \mathrm{~mm} \mathrm{Hg}$ (diastolic) in women and in men. There were 610 women and 116 men (Table 1). In the control group 489 patients of the entire group of 1,020 patients $(47.9 \%)$ had hypertension whilst 531 of the patients were normotensive. There were 410 women and 79 men. Parathyroid adenomas occurred with similar frequencies in the hypertensive and normotensive patients.

Hypertension appeared to be significantly more common in the PHPT $(72.1 \%$ in the case of women and $67.4 \%$ in the case of men) in comparison with our sex and age matched control group (women $45.9 \%$ and men 49.9\%) $(P<0.001)$. The estimated percentage of hypertensive patients with PHPT receiving pharmacological treatment was $64.5 \%$. It was slightly higher in females. This study has been drawn from our group of PHPT patients (Broulík et al., 2011).

There is statistically significant difference between BMI of all our patients with PHPT and our sex and age matched control group $\left(32.81 \pm 2.6 \mathrm{~kg} / \mathrm{m}^{2}\right.$ versus $\left.27.1 \pm 3.0 \mathrm{~kg} / \mathrm{m}^{2}\right)(P>0.01)$.

Serum uric acid levels were at baseline significantly higher in patients with PHPT when compared to controls. There were significant differences in mean serum uric acid levels between the whole group with PHPT $(368 \pm 54 \mu \mathrm{mol} / \mathrm{l})$ and the whole control group $(266 \pm 49 \mu \mathrm{mol} / \mathrm{l})(\mathrm{P}<0.01)$. It is again drawn from our group of PHPT patients (Broulík et al., 1987).

In the individual study cholelithiasis was proved in 168 of patients with primary hyperparathyroidism (25.89\%) of the entire group of 645 patients with PHPT. There were 157 women of the entire group of 518 women (30.3\%) and 11 men of the entire group of 127 men (8.66\%). Their mean age was $59.67 \pm 12$ years in women and $56.0 \pm 10$ years in men (Broulík et al., 2005).

In control group 314 patients of the entire group of 2,015 patients (15.6\%) had cholelithiasis. There were 260 women of the entire group of 1,505 women $(17.27 \%)$ and 54 men of the entire group of 510 men $(10.58 \%)$. Only in the case of women the difference is highly statistically significant $(P<0.001)$. The mean age of control patients was $64.55 \pm 13.8$ years in women and $61.2 \pm 12.4$ years in men. Drawn from our group of PHPT patients.

The diagnosis of acute pancreatitis has been based on a high serum amylase level and or abnormalities on ultrasound or computed tomography (CT) scan explorations, or ERCP (endoscopic retrograde cholangiopancreatography) examination. Only patients without biliary stones, hypertriglyceridemia and alcoholism were included in the pancreatitis group associated with PHPT. The incidence of acute pancreatitis is statistically higher $(4.85 \%)$ than in our control group 1.15\% $(P<0.05)$.

Again in the individual study (515 patients with PHPT) the diagnosis of stomach or duodenal ulcers has been based on X-ray or fibroscopic examinations. The patients with the chronic NSAID (nonsteroidal anti-inflammatory drugs) use have been excluded from the study. 
To determine the frequency of gastrointestinal symptoms in normal population we retrospectively analyzed 2,015 age and gender consecutive patients registered in outpatient ward in General University Hospital in Prague during the year 1998. Frequency was $4.3 \%$.

As the control data we have used also data from statistical department of our Ministry of Health for years 1997 (446 231 patients) and 1998 (432 495 patients) (0.4\%).

Gastrointestinal (GIT) complications of PHPT have been determined as being as a part of PHPT only when diagnosis of PHPT have been established usually as a high serum calcium and low phosphate, high ALP (alkaline phosphatase) and high iPTH. Peptic ulcers of various localizations were found in $15.28 \%$ of examined patients with PHPT which is higher incidence than in control group (4.3\%) $(\mathrm{P}<0.001)$.

The prevalence of diabetes mellitus in a series of 1,750 patients with proven PHPT was $24 \%$ which is significantly greater than that observed in nonhyperparathyroid control group (7\%).

\section{Discussion}

The clinical spectrum of primary hyperparathyroidism has undergone a continuous evolution since the classic description. There is still no definite explanation for the variability of the clinical manifestations of primary hyperparathyroidism. Up to about 40 years ago in former Czechoslovakia the disease was rarely diagnosed. It was characterized by marked hypercalcemia, recurrent renal stones and severe bone disease. The clinical presentation of PHPT has changed in the last 30 years and now the disease is characterized by hypercalcemia, less classic manifestation (bone and kidney) but the other nonclassic manifestation which belong to PHPT (Marcocci and Cetani, 2011).

From the period around 1981 the incidence of PHPT at the $3^{\text {rd }}$ Department of Medicine, First Faculty of Medicine, Charles University in Prague rose impressively for the identification of patients who were never diagnosed before. Most of the changes in the incidence of PHPT are explained by availability of PTH measurement and improving methods of localization. An elevated level of PTH with elevated calcium level indicates primary hyperparathyroidism.

The ratio women to men has not changed since 1981. However the incidence of PHPT increased in Czech Republic from the fifth to the seventh decade of life and the number of cases peaks in elderly women.

Nephrolithiasis still occurs in $31.4 \%$ of our patients with PHPT which is higher than in control group and also in European countries (5-15\%). Also the bone manifestation of hyperparathyroidism $(36.0 \%)$ of our patients is higher than in control group and in European countries (Silverberg and Bilezikian, 2001).

In the present large series of patients with clinically and biochemically proven primary hyperparathyrodism we found that $69.8 \%$ of patients have been suffering 
from hypertension. It has been suggested that arterial hypertension is common in PHPT and our data confirm this hypothesis. The $69.8 \%$ prevalence of hypertension between patients with PHPT is higher as compared with $47 \%$ prevalence of hypertension in general population in Czech Republic (Broulík et al., 1985); and also from our sex, age, BMI control group (48\%). The mean age of all our patients with PHPT at the time of diagnosis is $\mathbf{5 8 . 8}$ years which is almost the same like in general population (60 years) where prevalence of hypertension is $47 \%$ (Cífková et al., 2004).

Several pathogenetic mechanisms could be responsible for higher prevalence of hypertension in PHPT. Suggested mediators have included hypercalcemia, high PTH, plasma renin, renal functions, uric acid and BMI. Raised concentration of parathormone can be responsible for the hypertension. PTH was higher in our hypertensive patients with PHPT than in the normotensive patients with PHPT.The administration of parathyroid hormone stimulates the secretion of renin in dogs and in humans and is associated with an increase in plasma renin activity (Jorde et al., 2005). We have also shown raised plasma renin activity in healthy subjects without hypertension after administration of PTH (Broulík et al., 1986; Horký et al., 1986). Also the chronic elevation of parathormone in PHPT was accompanied by high plasma renin activity. After parathyroidectomy plasma renin activity levels fell to normal. The most plausible explanation may be the renal vasodilatory effect of PTH.A decrease in stretch of the afferent arterioles stimulates renin release from the juxtaglomerular apparatus. The patients with PHPT exhibit an impaired endothelial vasodilatory function and parathyroidectomy can reverse this defect.

Recent experimental and clinical evidence supports the possibility that an elevated uric acid level may lead to hypertension. Both hyperuricemia and gout occur with increased frequency in hyperparathyroidism and PHPT causes a reduced clearance of uric acid and raises the serum uric levels (Broulík et al., 1987). In our patients serum uric acid was higher in the PHPT patients than in control subjects.

We have found statistically significant changes in BMI between patients with PHPT and control group. Patients with PHPT are heavier reporting BMI than patients in control group.

Vitamin $D$ is fat soluble and is sequestered by adipose tissue. Thus increased body weight may promote vitamin $D$ deficiency, resulting in secondary hyperparathyroidism. Secondary hyperparathyroidism appears to increase the risk of developing parathyroid adenoma (Bolland et al., 2005).

There is certain relationship between primary hyperparathyroidism and crystal induced arthropathies. Calcium pyrophosphate deposition disease is a condition in which calcium pyrophosphate dihydrate crystals are deposited in joint articular cartilage, menisci and synovium. The main clinical presentations of calcium pyrophosphate dihydrate crystals are chondrocalcinosis-calcification of cartilage, pseudogout, acute joint inflammation due to crystal induced synovitis and pyrophosphate arthropathy-degenerative joint disease similar to osteoarthritis 
associated with calcium pyrophosphate crystal deposition (Vencovský and Broulík, 2004).

Compared to our control group it is evident that the incidence of gastric ulcers in PHPT is at least two-times higher. Hypercalcemia increases gastric acid secretion. While it might be difficult to prove it, it seems likely that association of peptic ulcer and pancreatitis with PHPT is more than coincidental and from our study it is obvious that significant association exists (Shearer and Imrie, 1986; Broulík et al., 2003).

A high prevalence of diabetes mellitus accompanying PHPT is now well established (Khaleeli et al., 2007). There is an experimental evidence that abnormal glucose metabolism in PHPT results from raised intracellular calcium that inhibits insulin stimulated glucose uptake thereby increasing insulin needs leading to diabetes mellitus (Škrha et al., 1988; Taylor and Khaleeli, 1997).

Localisation techniques include ultrasound, MRI (magnetic resonance imaging) and computerised axial tomography with the greatest reported success with use of 99 technetium labelled sestamibi single proton emission CT. Up to $89 \%$ of single parathyroid adenoma can be localized by this method (Kubinyi et al., 2010).

In the etiologic factors of PHPT the irradiation of the neck and upper chest for benign diseases is well-known risk factor for the development of PHPT however from our 1,750 patients we did not prove it. Only $2 \%$ of our patients with PHPT have a history of irradiation.

Surgical management of PHPT is a very effective method. The goal of treatment is to cure the disease by removing the abnormal parathyroid tissue. We recommend patients with symptomatic but also asymptomatic primary hyperparathyroidism for parathyroidectomy. Up to now we have team of surgeons which is well experienced and operate more than 200 patients with primary hyperparatyhroidism per year. Surgical treatment in hands of an experienced surgeon has $95 \%$ and higher success rate for permanent cure. From our studies there is growing evidence that suggests that PHPT is associated with increased incidence of hypertension, insulin resistance, dyslipidemia and cardiovascular disease. These considerations would argue in favour of intervention in asymptomatic PHPT with the hope of reducing life complication from these conditions. The result of operation is an improvement of health condition and resolution of symptoms. The number of patients who underwent surgical correction of PHPT in our country has been stable over the last decade but is still three times greater than that of the late 1970s.

Still, we cannot be satisfied, because many patients are still coming to the clinic with hyperparathyroidism in stage with already affected organs. Also, the time from the occurrence of the first difficulties or the appearance of hypercalcemia for a definitive diagnosis is still quite long.

\section{References}

Adami, S., Marcocci, C., Gatti, D. (2002) Epidemiology of primary hyperparathyroidism in Europe. J. Bone Miner. Res. 17, N18-N23. 
Bolland, M. J., Grey, A. B., Gamble, G. D., Reid, I. R. (2005) Association between primary hyperparahyroidism and increased body weight: a meta analysis. J. Clin. Endocrinol. Metab. 90, 1525-1530.

Broulik, P. D., Horký, K., Pacovský,V. (1985) Blood pressure in patients with primary hyperparathyroidism before and after parathyroidectomy. Exp. Clin. Endocrinol. 86, 346-352.

Broulík, P. D., Horký, K., Pacovský, V. (1986) Effect of parathyroid hormone on plasma renin activity in humans. Horm. Metab. Res. 18, 490-492.

Broulik, P. D., Štěpán, J. J., Pacovský,V. (1987) Primary hyperparathyrodism and hyperuricaemia are associated but not correlated with indicators of bone turnover. Clin. Chim. Acta 170, 195-200.

Broulík, P. D., Haas, T., Adámek, S. (2003) Further experience with peptic ulcer disease and pancreatitis as a diagnostic clue to primary hyperparathyroidism. Sb. Lek. 104, 247-254.

Broulík, P. D., Haas, T., Adámek, S. (2005) Analysis of 645 patients with primary hyperparathyroidism with special reference to cholelithiasis. Intern. Med. 44, 917-921.

Broulík, P. D., Broulíková, A., Adámek, S., Libánský, P., Tvrdoň, J., Broulíková, K., Kubinyi, J. (2011) Improvement of hypertension after parathyroidectomy of patients suffering from primary hyperparathyroidism. Int.J. Endocrinol. 2011, 309068, doi:10.1155/2011/309068.

Cífková, R., Škodová, Z., Lánská, V., Adámková, V., Novozámská, E., Petržilková, Z., Jozífková, M., Plášková, M., Hejl, Z., Palouš, D., Galovcová, M. (2004) Trends in blood pressure levels, prevalence, awareness, treatment, and control of hypertension in the Czech population from 1985 to 2000/01. J. Hypertens. 22, 1479-1485.

Feig, D. I., Kang, D. H., Johnson, R. J. (2008) Uric acid and cardiovascular risk. N. Engl. J. Med. 359, 1811-1821.

Horký, K., Broulík, P. D., Pacovský, V. (1986) The effect of parathyroid hormone on plasma renin activity in humans and hypertension in patients with primary hyperparathyrodism. J. Hypertens. 4, S585-S587.

Jorde, R., Svartberg, J., Sundsfjord, J. (2005) Serum parathyroid hormone as a predictor of increase in systolic blood pressure in men. J. Hypertens. 23, 1639-1644.

Khaleeli, A. A., Johnson, J. N., Taylor,W. H. (2007) Prevalence of glucose intolerance in primary hyperparathyroidism and the benefit of parathyroidectomy. Diabetes Metab. Res. Rev. 23, 43-48.

Kiernan, T. J., O'Flynn, A. M., McDermontt, J. H., Kearney, P. (2006) Primary hyperparathyroidism and the cardiovascular system. Int. J. Cardiol. 113, E89-E92.

Kubinyi, J., Trnka, J., Adámek, S., Libánský, P., Broulík, P. (2010) Clinical validation of a modified tomographic protocol for preoperative localization of parathyroid glands in patients with hyperparathyroidism based on volume rendering vizualization and 3D substraction. Eur. J. Nucl. Med. Mol. Imaging 37, S203-S204 (Suppl. 2).

Marcocci, C., Cetani, F. (2011) Primary hyperparathyroidism. N. Engl. J. Med. 22, 2389-2397.

Shearer, M. G., Imrie, C.W. (1986) Parathyroid hormone levels, hyperparathyroidism and acute pancreatitis. Br. J. Surg. 73, 282-284.

Silverberg, S. J., Bilezikian, J. P. (2001) Clinical presentation of primary hyperparathyroidism in the United States. In: The Parathyroids, $2^{\text {nd }}$ Edition, eds. Bilezikian, J. P., Marcus, R., Levine, M. A., Pp. 349-360, Raven Press, New York.

Škrha, J., Šrámková, J., Broulík, P., Páv, J. (1988) Glycoregulation in primary hyperparathyroidism. Sb. Lek. 90, 200-205. (in Czech)

Taylor, W. H., Khaleeli, A. A. (1997) Prevalence of primary hyperparathyroidism in patients with diabetes mellitus. Diabet. Med. 14, 386-389.

Vencovský, J., Broulík, P. (2004) The endocrine system. In: Oxford Textbook of Rheumatology. Isenberg, D. A., Maddison, P. J., Woo, P., Glass, D., Breedveld, F. C., Oxford University Press, Oxford. 